\title{
Self-reported concussion history: impact of providing a definition of concussion
}

This article was published in the following Dove Press journal:

Open Access Journal of Sports Medicine

7 May 2014

Number of times this article has been viewed

\author{
Clifford A Robbins' \\ Daniel H Daneshvar ${ }^{1,2}$ \\ John D Picano ${ }^{1,3}$ \\ Brandon E Gavett ${ }^{1,4}$ \\ Christine M Baugh ${ }^{1,2}$ \\ David O Riley' \\ Christopher J Nowinski ${ }^{1,2,5}$ \\ Ann C McKee ${ }^{1,2,6-8}$ \\ Robert C Cantu',5,9,10 \\ Robert A Stern ${ }^{1,2,8,9}$ \\ 'Center for the Study of Traumatic \\ Encephalopathy, ${ }^{2}$ Department of \\ Neurology, Boston University School \\ of Medicine, Boston, MA, USA; ${ }^{3}$ School \\ of Medicine and Biomedical Sciences, \\ University of Buffalo, Buffalo, NY, USA; \\ ${ }^{4}$ Department of Psychology, University \\ of Colorado, Colorado Springs, \\ CO, USA; ${ }^{5}$ Sports Legacy Institute, \\ Waltham MA, USA; ${ }^{\circ}$ United States \\ Department of Veterans Affairs, VA \\ Boston Healthcare System, Boston, \\ MA, USA; 'Department of Pathology, \\ ${ }^{8}$ Alzheimer's Disease Center, \\ 'Department of Neurosurgery, \\ Boston University School of Medicine, \\ Boston, MA, USA; ${ }^{10}$ Department of \\ Neurosurgery, Emerson Hospital, \\ Concord, MA, USA
}

Background: In recent years, the understanding of concussion has evolved in the research and medical communities to include more subtle and transient symptoms. The accepted definition of concussion in these communities has reflected this change. However, it is unclear whether this shift is also reflected in the understanding of the athletic community.

What is known about the subject: Self-reported concussion history is an inaccurate assessment of someone's lifetime exposure to concussive brain trauma. However, unfortunately, in many cases it is the only available tool.

Hypothesis/purpose: We hypothesize that athletes' self-reported concussion histories will be significantly greater after reading them the current definition of concussion, relative to the reporting when no definition was provided. An increase from baseline to post-definition response will suggest that athletes are unaware of the currently accepted medical definition.

Study design: Cross-sectional study of 472 current and former athletes.

Methods: Investigators conducted structured telephone interviews with current and former athletes between January 2010 and January 2013, asking participants to report how many concussions they had received in their lives. Interviewers then read participants a current definition of concussion, and asked them to re-estimate based on that definition.

Results: The two estimates were significantly different (Wilcoxon signed rank test: $z=15.636$, $P<0.001)$. Comparison of the baseline and post-definition medians ( 7 and 15 , respectively) indicated that the post-definition estimate was approximately twice the baseline. Follow-up analyses indicated that this effect was consistent across all levels of competition examined and across type of sport (contact versus non-contact).

Conclusion: Our results indicate that athletes' current understandings of concussions are not consistent with a currently accepted medical definition. We strongly recommend that clinicians and researchers preface requests for self-reported concussion history with a definition. In addition, it is extremely important that researchers report the definition they used in published manuscripts of their work.

What this study adds to existing knowledge: Our study shows that unprompted reporting of concussion history produces results that are significantly different from those provided after a definition has been given, suggesting one possible mechanism to improve the reliability of self-reported concussion history across multiple individuals.

Keywords: concussion, self-report, sports-related concussion

\section{Introduction}

The Centers for Disease Control and Prevention estimate that between 1.6 and 3.8 million sports-related concussive injuries occur annually in the United States. ${ }^{1,2}$ These types of injuries have been implicated in a variety of long-term neurological 
consequences, including potential long-term behavioral changes, ${ }^{3}$ Post-Concussion Syndrome, ${ }^{4}$ Chronic Traumatic Encephalopathy, ${ }^{5-7}$ and Chronic Traumatic Encephalopathy with Motor Neuron Disease. ${ }^{6,8}$ The medical community has revised the definition of concussion several times over the last few decades to better categorize injuries that contribute to these various sequelae. It is unclear, however, whether these changes have been understood by the athletic community. Our investigation seeks to evaluate whether a "disconnect" exists between athletes' understanding of concussion and a current definition accepted by the medical and scientific community.

Early definitions of concussions characterized them as injuries with relatively severe and long-lasting symptoms. In 1965, the Ad Hoc Committee to Study Head Injury Nomenclature suggested a graded system centered around loss of consciousness less than or greater than 5 minutes. ${ }^{9}$ Cantu later revised that system to incorporate post-traumatic amnesia as an indicator of concussion, ${ }^{10}$ which was further revised by Kelly et $\mathrm{al}^{11}$ to include general confusion as a potential symptom. In 2008, the Third International Conference on Concussion in Sport expanded the definition of concussion to include a wide range of potential indicators, including headaches, visual disturbances, nausea, dizziness, sleep disturbances, and memory problems. ${ }^{12}$ In addition, members of the conference stressed that an even relatively brief presentation of any single symptom could be indicative of a concussion. ${ }^{12}$ Definitions based on this framework have appeared several times in the recent literature. ${ }^{13-15}$

Despite increases in concussion education of current athletes by state legislatures, ${ }^{16}$ the National Collegiate Athletic Association, ${ }^{17}$ and professional sports leagues, ${ }^{18}$ and increase in awareness of concussions among the general public as a result of increased media attention, it is unclear whether this shift in understanding of the definition of concussion has disseminated to current and former athletes. Considering that retrospective self-reported concussion history is an extremely important tool for both clinicians and researchers, it is important that both current and former athletes understand the current definition of concussion so that both the athlete and clinician are referring to the same phenomenon when considering concussion history. The current study seeks to evaluate whether current and former athletes are basing their self-reported concussion history on a currently accepted definition of concussion by asking them to report their history unprompted, and then again after hearing a current definition. We hypothesize that, despite increased media attention to sports-related concussion, athletes are unaware of the currently accepted definition of concussion.

\section{Methods}

We conducted telephone-based structured interviews as part of a cross-sectional study of former and current athletes participating in the Longitudinal Evaluation to Gain Evidence of Neurodegenerative Disease (LEGEND) ${ }^{15}$ project at the Boston University Center for the Study of Traumatic Encephalopathy (CSTE). Participants were considered athletes if they had a history of participating in at least one organized sport featuring officiated competition. Participants' primary sports are reported in Table 1 . Interviews were conducted by trained research assistants. Informed consent was provided in accordance with the Boston University School of Medicine Institutional Review Board. All participants volunteered to take part in the study after contacting the CSTE; investigators did not actively recruit subjects for this study. Further discussion of the LEGEND study protocol has been reported elsewhere in the literature. ${ }^{15}$ Participants were asked to provide an estimate of the number of concussions they had sustained throughout their lives. No guidance or additional information was provided by the interviewer. After participants had provided their estimated number, interviewers read the following definition, based on the Centers for Disease Control ${ }^{1}$ statement on sports-related concussion and the consensus statement on concussion in sport from

Table I Primary sport frequency

\begin{tabular}{ll}
\hline Primary sport & Frequency \\
\hline Football & 224 \\
Ice hockey & 53 \\
Soccer & 38 \\
Rugby & 26 \\
Baseball & 21 \\
Basketball & 19 \\
Entertainment wrestling & 16 \\
Boxing & 10 \\
Amateur wrestling & 9 \\
Equestrian & 6 \\
Lacrosse & 6 \\
Track and field & 5 \\
Swimming & 5 \\
Cycling & 4 \\
Tennis & 3 \\
Sports with two participants* & 3 \\
Sports with one participant** & 8 \\
Total & 15 \\
\hline
\end{tabular}

Notes: *Sports with two participants: auto racing, motorcycle racing, skiing, softball; **Sports with one participant: bowling, cross country running, diving, field hockey, golf, gymnastics, horse jumping, karate," ice skating, inline skating, martial arts," parachuting, roller hockey, snowboarding, volleyball; "contact sport for purposes of analysis. 
the Third International Conference on Concussion in Sport held in Zurich: ${ }^{12}$

Some people have the misconception that concussions only happen when you black out after a hit to the head or when the symptoms last for a while. But, in reality, a concussion has occurred anytime you have had a blow to the head that caused you to have symptoms for any amount of time. These include: blurred or double vision, seeing stars, sensitivity to light or noise, headache, dizziness or balance problems, nausea, vomiting, trouble sleeping, fatigue, confusion, difficulty remembering, difficulty concentrating, or loss of consciousness. Whenever anyone gets a ding or their bell rung, that too is a concussion. ${ }^{15}$

Interviewers then prompted participants to again estimate their lifetime number of concussions, but based on the above definition. Since the project was conducted, a more recent consensus statement on concussion in sport has been created, which remains consistent with our definition. ${ }^{13}$ Baseline and post-definition concussion history estimates were analyzed in PSAW V.20 (IBM, Armonk, NY, USA).

\section{Results}

Baseline and post-definition concussion history estimates from 472 athletes (417 males) between the ages of 20 and 85 years (mean 44 years, standard deviation \pm 14 years) were analyzed. Six participants were excluded due to incomplete data. The data were highly skewed (baseline skewness 18.6, post-definition skewness 15.1), prohibiting the use of parametric statistical techniques. We, therefore, used Wilcoxon's signed-rank test to determine if there was a statistical difference between the two estimates. The difference between the two estimates was significant ( $z=15.6, P<0.001$ ). To ensure that the effect was not driven by outliers, we eliminated four records whose baseline or post-definition responses fell three standard deviations above or below their respective means and re-ran the test. The result remained significant ( $z=15.6$, $P<0.001)$. Three hundred and forty-one (341) participants $(73 \%)$ increased their estimate from baseline to postdefinition, while $118(25 \%)$ maintained the same estimate and six $(2 \%)$ decreased their estimate. To determine the direction and strength of the effect, we compared the median responses from the baseline and post-definition responses. The medians were separated by approximately a factor of 2 (baseline 7, post-definition 15), indicating that the post-definition estimate was approximately twice the baseline.

We further analyzed baseline to post-definition estimates in contact vs non-contact athletes. If a participant listed a contact sport as their primary sport, we categorized them as a contact athlete (see Table 1 for a list of contact sports). We ran Wilcoxon signed-rank tests for contact athletes $(z=7.464, P<0.001)$ and non-contact athletes $(z=13.485$, $P<0.001$ ), suggesting that both contact and non-contact athletes were unaware of the current definition of concussion.

To determine if this effect was driven by the level at which our athletes competed, we split the file into athletes who only competed in high school or below, those who competed in college, and those who competed professionally. We ran additional Wilcoxon signed-rank tests for each group. All were significant, (High school $[\mathrm{N}=88]$ : $z=6.116, P<0.001$; College [N=202]: $z=10.496, P<0.001$; Professional [N=143]: $z=8.860, P<0.001)$ suggesting that this was not an effect driven by solely those who competed at higher levels.

\section{Discussion}

The current investigation is the first, to our knowledge, to quantify the disparity between athletes' personal understandings of what a concussion is and a medically accepted definition. Relative to their baseline estimates, our participants reported significantly more concussions after interviewers read a currently accepted definition of concussion. Further, the vast majority of our participants increased their estimates frombaseline to post-definition. This pattern remained consistent across levels of competition, and across contact versus non-contact athletes. Our findings indicate that our participants had a different and more exclusionary idea of what a concussion is, possibly due to the fact that during the time when they played organized sports the prevailing definition described concussions as exhibiting more severe symptoms. This effect may have been influenced by demand characteristics, or a tendency of participants to over-report in an attempt to "help" the researchers when asked a second time. It is worth noting, however, that a quarter of the sample did not change their estimate, suggesting that this potential confound was not unilateral.

Our results have important implications in several domains. First, our findings should be considered in the management of sports-related concussions. The current guideline for preseason management of sports-related concussions, according to the American Medical Society for Sports Medicine, relies in part on self-report history of concussion. ${ }^{13}$ In light of our findings, it is suggested that clinicians provide athletes with a definition of a concussion before querying their concussion histories. Future research could expand on our findings by investigating the effectiveness of providing a definition in different formats. 
Our findings also have very important implications in the field of concussion research. Currently, retrospective self-reported concussion history is, in many cases, the only available estimate of lifetime exposure. Several studies have cast doubt on the accuracy of this type of estimate. Kerr et $\mathrm{al}^{19}$ examined self-reported concussion histories taken from retired National Football League (NFL) players by survey once in 2001 and again in 2010, to assess how reliable they were over time. Despite using a definition-prompting paradigm similar to our post-definition procedure, they found that these two estimates were only moderately related (Cohen's $k=0.485$ ). Considering the limitations of this metric, it is critical that researchers make efforts to eliminate any known biases in future research. In an unprompted paradigm, participants may estimate their concussion histories using a variety of potential definitions. By systematically providing the same definition to all participants before they estimate, researchers eliminate the variance stemming from differential understanding of concussions and are left only with variability associated with self-report. We strongly recommend that, in future research, all papers both use a definition before collecting self-reported concussion history and report the definition used in the manuscript. Several recent papers have used self-reported concussion history estimates as covariates for neuroimaging findings; however, it is unclear from the published manuscripts what, if any, definition was used to prompt self-reported concussion history. Because of the lack of a reported definition, the validity of these results is called into question.

The importance of this finding is not limited to evaluating the concussion history in those who have experienced concussions: it is also important in helping to confirm a lack of concussion history in those who have not. In our sample of 472 athletes, 17 reported no concussions at baseline. After reading the definition, only four maintained that estimate. This highlights the importance of providing all participants in a research study with a definition of concussion. An investigation that uses self-reported concussion history to define a group as having no history of concussion but fails to provide a definition to control participants runs the risk of inappropriately including participants unaware of their own concussion history.

Our results also have encouraging implications in the arena of concussion education. Our definition took less than 30 seconds to administer over the phone, and it was sufficiently powerful to significantly alter our participants' estimates of their lifetime exposure. Previous reports have shown that a 30-minute concussion education program administered to young athletes can help them better recognize the symptoms of a concussion..$^{20}$ Our results suggest that simply providing a current definition of a concussion to at-risk populations may help increase proper identification of these types of injuries through recognition of concussion symptoms. Future research should aim to assess how robust this education effect is over time, and whether it can have effects comparable to those of more time- and resourceintensive concussion education programs.

This study has several limitations. First, our sample was not recruited from a specific cohort; all participants sought out our center looking for research on the effects of concussions. This could have produced a bias in our sample such that individuals who have suffered more concussions were more likely to participate, or that individuals who knew less about the effects of concussions were more likely to reach out in order to learn more. Future research should seek to assess understanding in a more unbiased sample of athletes. It is worth noting, however, that even individuals who are motivated enough to seek out and participate in concussion-specific research studies do not have a clear understanding of what a concussion is. Our sample is also heavily male. Considering the differences in concussion reporting between male and female athletes, ${ }^{20}$ future research should seek to evaluate the understanding of concussion in a female population. Our participants are also predominantly former athletes (mean age 44 years) who played their sports when concussion diagnoses were reserved for injuries with more severe symptoms. Future research could use a paradigm similar to ours in a sample of athletes who are either currently playing or who played recently when the prevailing concussion definition was closer to what it is today. Finally, the majority of our participants played football as their primary sport; it is possible that athletes in other sports could be more or less aware of the definition of concussion relative to our sample. Our finding that both contact and non-contact athletes increased their concussion estimates post-definition suggests that our findings were not solely driven by differences in sport.

In summary, this study indicates that researchers and clinicians cannot assume that athletes have a clear understanding of the definition of concussion. It is necessary, therefore, to prompt athletes with a current definition when asking about their concussion histories. In a research setting, it is also critical that the definition used be reported in the published findings.

\section{Disclosure}

The authors report no conflicts of interest in this work. 


\section{References}

1. http://www.cdc.gov [homepage on the Internet]. Concussion in Sports. Centers for Disease Control and Prevention, Atlanta, GA; Concussion in Sports. 2013 [updated July 22, 2013]. Available from: http://www. cdc.gov/concussion/sports/. Accessed February 28, 2014.

2. Langlois JA, Rutland-Brown W, Wald MM. The epidemiology and impact of traumatic brain injury: a brief overview. $J$ Head Trauma Rehabil. 2006;21(5):375-378.

3. Daneshvar DH, Riley DO, Nowinski CJ, McKee AC, Stern RA, Cantu RC. Long-term consequences: Effects on normal development profile after concussion. Phys Med Rehabil Clin N Am. 2011;22(4):683-700.

4. Cantu RC, Herring SA, Putukian M. Concussion. N Engl J Med. 2007;356:1787-1789.

5. McKee AC, Cantu RC, Nowinski CJ, et al. Chronic traumatic encephalopathy in athletes: progressive tauopathy after repetitive head injury. J Neuropathol Exp Neurol. 2009;68(7):709-735.

6. McKee AC, Gavett BE, Stern RA, et al. TDP-43 proteinopathy and motor neuron disease in chronic traumatic encephalopathy. J Neuropathol Exp Neurol. 2010;69(9):918-929.

7. Stern RA, DaneshvarDH, Baugh CM, et al. Clinical presentation of chronic traumatic encephalopathy. Neurology. 2013;81(13):1122-1129.

8. McKee AM, Stern RA, Nowinski CJ, et al. The spectrum of disease in chronic traumatic encephalopathy. Brain. 2013;136(Pt1):43-64.

9. Congress of Neurological Surgeons: Proceedings of the Congress of Neurological Surgeons in 1964: Report of the Ad Hoc Committee to Study Head Injury Nomenclature. Clin Neurosurg. 1966;12:386-394.

10. Cantu RC. Guidelines for return to contact sports after cerebral concussion. Physician Sportsmed. 1986;14(10):75-83.

11. Kelly J, Nichols JS, Filley CM, Lillehei KO, Rubinstein D, Kleinchmidt-DeMasters BK. Concussion in Sports: Guidelines for the Prevention of Catastrophic Outcomes. JAMA. 1991;266:2867-2869.

12. McCrory P, Meeuwisse W, Johnston K, et al. Consensus statement on concussion in sport - the third international conference on concussion in sport held in Zurich, Nov 2008. Phys Sportsmed. 2009;37(2):141-159.
13. Harmon KG, Drezner JA, Gammons M, et al. American Medical Society for sports medicine position statement: Concussion in sport. Clin J Sport Med. 2013;23(1):1-18.

14. McCrory P, Meeuwisse W, Aubry M, et al. Consensus statement on concussion in sport - the fourth international conference on concussion in sport held in Zurich, Nov 2012. Phys Ther Sport. 2013;14(2): e1-e13.

15. Seichepine DR, Stamm JM, Daneshvar DH, et al. Profile of self-reported problems with executive functioning in college and professional football players. J Neurotrauma. 2013;30(14):1299-1304.

16. NFL [webpage on the Internet]. The Zachary Lystedt Law. 2012. Available from: http://www.nflevolution.com/article/The-Zackery-LystedtLaw?ref=270. Accessed February 28, 2014.

17. NCAA [homepage on the Internet]. Concussion Management Plan Memorandum. NCAA; 2010. Available from: http://www.ncaa.org/ wps/wcm/connect/327bf600424d263692cdd6132e10b8df/Memo+C oncussion+Managment+04292010.pdf?MOD=AJPERES\&CACHE ID=327bf600424d263692cdd6132e10b8df. Accessed February 28, 2014.

18. NFL [homepage on the Internet]. New NFL rules designed to limit head injuries. NFL; 2010 [published August 6, 2010; updated July 26, 2012]. Available from: http://www.nfl.com/news/story/09000d5d81990bdf/ article/new-nfl-rules-designed-to-limit-head-injuries. Accessed February 28, 2014.

19. Kerr ZY, Marshall SW, Guskiewicz KM. Reliability of concussion history in former professional football players. Med Sci Sports Exerc. 2012;44(3):377-382.

20. Bagley AF, Daneshvar DH, Schanker BD, et al. Effectiveness of the SLICE program for youth concussion education. Clin J Sport Med. 2012;22:385-389. PMID: 22929042.

\section{Publish your work in this journal}

Open Access Journal of Sports Medicine is an international, peer-reviewed, open access journal publishing original research, reports, reviews and commentaries on all areas of sports medicine. The manuscript management system is completely online and includes a very quick and fair peer-review system.

\section{Dovepress}

Visit http://www.dovepress.com/testimonials.php to read real quotes from published authors. 